\title{
CONCEPCIONES DE LOS PROFESORES SOBRE LA ENSEÑANZA DE LAS ECUACIONES DIFERENCIALES A ESTUDIANTES DE QUÍMICA Y BIOLOGÍA. ESTUDIO DE CASOS
}

\author{
MORENO, M. ${ }^{1}$ y AZCÁRATE, C. ${ }^{2}$ \\ 1 Departament de Matemàtica. Universitat de Lleida. \\ ${ }^{2}$ Departament de Didàctica de les Matemàtiques $i$ les Ciències Experimentals. \\ Universitat Autònoma de Barcelona.
}

\begin{abstract}
SUMMARY
Intending to quest about the conceptions math teachers hold about how to teach Differential Equations to chemistry and biology students, we have devised a research tool which allows us to derive relevant information. We use different means to collect the adequate data related to the qualitative research, targeting the exploration of what teachers «say they do» and what «they do and would like to do». The use of concept maps and a questionnaire, along with a recorded interview, has revealed itself as an accurate means for the appropriate analysis of data, as shown in the case study we hereby include.
\end{abstract}

\section{INTRODUCCIÓN}

La preocupación por la enseñanza de las matemáticas a nivel avanzado y los estudios de Artigue (1989) sobre la importancia que las ecuaciones diferenciales tienen en Ios contenidos de los primeros cursos universitarios en carreras científico-técnicas y su relación con las nuevas tecnologías, nos han llevado a investigar y reflexionar sobre aspectos ligados directamente con el proceso de enseñanza de las ecuaciones diferenciales, tales como: concepciones de los profesores, actitudes y creencias, discurso del profesor, etc., en estudios científico-experimentales como química y biología.

Un estudio preliminar sobre el tema (Moreno, 1995) evidenció ciertas limitaciones desde el punto de vista de la formación del profesor universitario a la hora de hacer una propuesta coherente con los intereses, expectativas y necesidades de los estudiantes de estas carreras científico-experimentales, reconociendo que generalmente la asignatura de ecuaciones diferenciales destinada a químicos y biólogos difería de la correspondiente para matemáticos y físicos, en tan sólo el grado de exigencia.

El carácter interdisciplinar de las ecuaciones diferenciales, así como el hecho de ser no sólo objeto de conocimiento, sino además un instrumento para los futuros químicos y biólogos, nos hizo pensar si realmente no debían tenerse en cuenta, de forma especial, a los destinatarios de la asignatura, así como sus futuras necesidades profesionales. Todas estas consideraciones junto con el aparente «inmovilismo» al que parece estar sujeta la enseñanza de las ecuaciones diferenciales, manteniéndose al margen de las nuevas tecnologías, fueron el principal motor de este estudio que presentamos a continuación.

De esta forma diseñamos una investigacion que nos permitiera conocer, con cierto detalle, las ideas de los profesores de matemáticas al respecto, así como identi- 
ficar las dificultades y necesidades en relación con la enseñanza de las ecuaciones diferenciales en estas licenciaturas. Así, los centros de interés particulares en torno a los cuales articulamos el estudio se concretan en estos dos puntos:

a) Indagar acerca de las concepciones de los profesores de matemáticas sobre la enseñanza de las ecuaciones diferenciales en carreras científico-experimentales.

b) Detectar y descubrir dificultades relativas al «qué" y «cómo» enseñar las ecuaciones diferenciales en dichas titulaciones.

\section{MARCO TEÓRICO}

Desde la perspectiva del filtro del profesor y atendiendo a las preguntas: ¿a quién se enseña?, ¿quiến enseña? y ¿cómo y qué enseñar?, se configuró un marco teórico como modelo que nos permitiera explicar los hechos detectados, observados y descubiertos.

Al analizar las concepciones de los profesores de matemáticas sobre el aprendizaje de las ectuaciones diferenciales, hemos considerado relevante la teoría cognitiva desarrollada por los profesores Tall y Dreyfus en relación con el desarrollo y crecimiento del pensamiento matemático avanzado. Así, hemos concedido especial importancia a la noción de esquema conceptual (concept image) desarroliada por Tall y Vinner (1981), posteriormente perfilada y matizada por Vinner y Dreyfus (1989), Dreyfus (1990) y revelada útil por las investigaciones de Azcárate $(1990,1992)$ acerca de las nociones de pendiente de Ia recta y derivada. La noción de esquema conceptual nos parece una buena forma de expresar la estructura cognitiva del individuo, en cuanto a sus imágenes mentales, representaciones, propiedades, procedimientos, ejemplos, contraejemplos, etc., asociados a un concepto matemático. Parte de su importancia en nuestro estudio reside en detectar si el profesor potencia el establecimiento de conexiones entre esquemas conceptuales existentes y, con ello, favorece la flexibilidad mental, base del éxito del crecimiento cognitivo.

Asimismo, hemos recogido las aportaciones de Dubinsky y Lewin (1986), Sfard (1987), Dreyfus (1990) y Sfard (1991) en torno a la idea de dualidad procesoconcepto $\mathrm{y}$, sobre todo, en torno a la noción de procept desarrollada por Tall (1994a, 1994b, 1995).

Un procepto elemental es la amalgama de un proceso, un concepto relativo a éste y producido por ese proceso y un símbolo que representa ambos, el proceso y el concepto. Un procepto consiste en una colección de proceptos elementales que tienen el mismo objeto. [...] es una noción cognitiva, no matemática; cualquiera podría estar tentado de definir un "procepto" elemental como una tripla ordenada (proceso, concepto y símbolo) y un procepto como una clase de cquivalencia de ternas ordenadas que tienen el mismo objeto.

(Tall, 1994a).
En nuestro estudio todo ello adquicre una importancia fundamental, al permitimos analizar qué tratamiento del concepto matemático «ecuación diferencial» ponen en práctica los profesores de matemáticas participantes en el estudio.

Además, las concepciones de los profesores están impregnadas de sus ideas personales, creencias, etc. sobre su papel como docentes, la materia objeto de enseñanza y los estudiantes a los cuales va dirigida ésta, por lo que no podemos olvidar en el marco teórico del «pensamiento del profesor» un análisis de aspectos tales como: toma de decisiones, planificación, sistema de creencias, actitudes personales. Hemos establecido lo que hemos llamado «estilos docentes», que nos permitirán valorar la influencia de estos factores en su actuación como docentes en el marco de una enseñanza universitaria a estudiantes, futuros químicos y biologos, y que ponen de manifiesto que el profesor está sujeto a influencias externas e internas que condicionan y delimitan su actuacion.

Finalmente, y en el caso de un estudio centrado en el profesor, no debemos olvidar los aspectos directamente ligados con la gestión de aula, que son los que regulan las interacciones con el estudiante y con la materia, en cuanto al tipo de tarea propuesta, los recursos utilizados, etc.

Por lo tanto, situamos nuestro trabajo en el marco de estas investigaciones y nos apoyamos en él para tratar de explicar los resultados obtenidos de los distintos instrumentos usados para la investigacion.

\section{METODOLOGÍA DE INVESTIGACIÓN}

La metodología seguida en el estudio es cualitativa, de naturaleza descriptiva, holística y exploratoria. A partir de un estudio de cuatro casos hemos abarcado el fenómeno de estudio de las concepciones de los profesores y gestión del aula, sobre la enseñanza de las ecuaciones diferenciales, considerando gran parte de los aspectos relacionados con éste.

Teniendo en cuenta que la información a partir de la cual hemos reflexionado y sacado conclusiones procede de las opintones y puntos de vista de los cuatro profesores participantes en el estudio, decidimos usar tres instrumentos de recogida de datos, con el fin de «validar» la información que nos proporcionaba cada uno de ellos (Miles y Hubermann, 1987). Así, los datos que hemos tenido en cuenta para este estudio proceden de los mapas conceptuales elaborados por cada profesor, de un cuestionario con preguntas abiertas y cerradas, y de una entrevista grabada.

Los participantes del estudio son cuatro matemáticos de distintas universidades de España. El profesor «A» da clase en la facultad de químicas desde hace dieciséts años y ha compaginado su actividad docente con su actividad investigadora en temas relacionados con la matemática aplicada a Ia industria. El profesor «B» da 
clase a un grupo de la facultad de químicas y a dos grupos más en la facultad de matemáticas, en asignaturas de geometría y topología; lleva seis años impartiendo docencia en la universidad, aunque durante catorce, aproximadamente, ha sido profesor de enseñanza media. El profesor $« C$ da igualmente clase en las facultades de química y matemáticas, lleva cuatro años en la universidad y su actividad funđamental está relacionada con topología. Finalmente, el profesor «D» da clases en la facultad de biología, lleva veinte años ligado al mundo universitario y toda su docencia en esta facultad, desde aproximadamente catorce años, es en relación con la estadística y matemáticas para biólogos.

Los instrumentos de recogida de datos destinados a los cuatro profesores de la muestra fueron:

a) Un mapa conceptual sobre jas concepciones de jos profesores acerca de la enseñanza de las ecuaciones diferenciales a estudiantes de química o biología. La actividad concreta que se pedía a los participantes del estudio fue construir un mapa conceptual sobre el tema EDO como asignatura para los estudiantes de química o biología. Para facilitar la elaboración de éste, se envió un dossier con información sobre mapas conceptuales y ejemplos provenientes de diferentes áreas del saber (como sociología, historia, biología, etc.), distintas de las matemáticas, con el fin đe no condicionar la elaboración del mapa conceptual personal de cada uno de los profesores; además se les propuso, como actividad previa a la elaboración del mapa, la confección de una lista de palabras relacionadas y sugeridas por el tema, de forma que les ayudara a la definitiva elaboración de «su mapa conceptuals.

b) Un cuestionario con cuatro cuestiones seleccionadas de entre problemas de exámenes proporcionados por los profesores «A» y «B». En estas cuestiones se pide a los profesores reflexionar acerca de un problesna de modelización, dos tipos diferentes de ecuaciones diferenciales de primer y segundo orden, resolubles por métodos diferentes, y un sistema de ecuaciones diferenciales. A su vez, y para guiar la reflexión de los profesores, en cada cuestión se plantearon preguntas abiertas y cerradas sobre aspectos tales como: valorar los diferentes métodos de resolución de ecuaciones y sistemas diferenciales desde el punto de vista matemático y desde el interés científico para un químico y biólogo, valorar la importancia e interés histórico del método del factor integrante para la resolución de determinadas ecuaciones diferenciales, proponer problemas reales del ámbito de la química y biología que pudieran modelizarse a partir de ecuaciones diferenciales de segundo orden y de sistemas de ecuaciones diferenciales no autónomos, valorar otros métodos de resolución distintos a los que se explicitan en el cuestionario y que pudieran resultar más significativos para los estudiantes, etc.

c) Una entrevista grabada que se realizó tras la elaboración del mapa conceptual y la entrega del cuestionario. Se trataba de una entrevista cerrada que constaba de tres momentos claramente diferenciados. Uno inicial en el que se pedía a cada profesor explicar su mapa conceptual, la estructura general de éste, el significado de ciertas proposiciones no demasiado claras, etc. Otro momento de Ia entrevista se destinó a matizar las respuestas del cuestionario y argumentar sus respuestas. Finalmente, se pidió a los profesores su opinión sobre la gestión de clase, el tipo de actividades de aprendizaje, su postura personal frente a las nuevas tecnologias, etc. La entrevista tuvo una dura* ción aproximada de una hora y media.

El propósito del mapa conceptual era recoger información de los modelos cognitivos de los profesores sobre la enseñanza de las ecuaciones diferenciales, y tratar de captar el significado y la estructura de los conocimientos que el profesor transmite a los estudiantes. El propósito del cuestionario era conocer el punto de vista de los profesores acerca de aspectos metodológicos, concep. tuales y procedimentales de la materia en sí misma; asimismo, consideramos el cuestionario como un complemento del mapa conceptual. El propósito de la entrevista fue matizar y aclarar aspectos del mapa conceptual y del cuestionario, que no quedaron lo suficientemente claros, con el fin de evitar una inadecuada interpretación de los datos recogidos.

Estos instrumentos se conjugaron con el análisis de los programas de la asignatura, materiales de aula, enunciados de exámenes y los libros de texto recomendados para el uso de los estudiantes. Este análisis trataba de establecer las posibles relaciones existentes entre lo que cada profesor decía que hacía y cómo concebía la asignatura (datos procedentes principalmente de los tres instrumentos de investigación ya descritos), y lo que en la práctica evidenciaban sus decisiones en relación con el material, ejercicios, etc.

\section{ANÁLISIS DE LOS DATOS}

\section{Análisis en general}

En el análisis de los datos se tuvo muy en cuenta la entrevista grabada. Por un lado, se realizó un análisis del mapa conceptual de cada profesor apoyado en sus explicaciones grabadas y, por el otro, el anátisis de los cuestionarios, pregunta por pregunta, apoyándonos en la entrevista grabada para explicitar matices que no quedaron claros en las respuestas de aquél.

Finalmente, tratamos de hacer un análisis conjunto que pretendía aunar características propias de cada profesor procedentes de todas las fuentes de datos de que disponíamos. El análisis, aunque largo y tedioso, resultó ser muy fructífero y rico en detalles.

\section{Análisis en particular}

\section{a) Análisis del mapa conceptual}

Dado que nuestro referente en el uso de los mapas conceptuales como instrumento de investigación lo situamos en Novak y Gowin (1988), y debido a que el 
análisis de los datos que proponen estos autores no nos resultaba válido para nuestro propósito, decidimos diseñar nuestro propio instrumento de análisis cualitativo de los mapas conceptuales, el cual consistió en un análisis apoyado en la entrevista grabada y elaborado en dos niveles: microanálisis y macroanálisis.

El microanálisis consiste en un análisis fino y en detalle basado en la interpretación, paso a paso, del mapa conceptual. Así, se pidió a los profesores que explicaran el significado particular que habían otorgado a las proposiciones que aparecían en su mapa conceptual, que justificaran las relaciones cruzadas que establecían entre los conceptos, que explicitaran los nexos entre conceptos que no siempre estaban presentes, que establecieran niveles de jerarquía si es que existían, etc.

El macroanalisis trataba de agrupar términos que englobaran conceptos secundarios o derivados de los principales y que no aportaban información nueva a la ya aportada por los primeros. A partir de la estructura conceptual inicial, este análisis nos permitió considerar otras estructuras conceptuales más generales y que proporcionaban una visión más global de las concepciones de cada profesor.

\section{b) Análisis del cuestionario}

Las preguntas cerradas se valoraron de 1 a 5 . Para facilitar la interpretación de las respuestas de los profesores se usó una escala como la que sigue:

\section{$1+{ }^{2}-{ }^{3}-{ }^{4}-5$ \\ POCO NORMAL MUCHO}

Para facilitar el análisis de los ítems y relacionarlo con los objetivos específicos del estudio, los agrupamos en cuatro caracteres: métodos de resolución de ecuaciones diferenciales, objetivo y tipo de actividad propuesta, conocimiento y aprovechamiento de la historia, uso de nuevas tecnologias.

Las preguntas abiertas permitieron hacernos idea del grado de flexibilidad y capacidad de los profesores para buscar situaciones conflictivas e interesantes que provocaran actitudes de búsqueda en los estudiantes.

El análisis de cada pregunta se realizó en dos niveles: descriptivo global y descriptivo particular. En el primero, se explicitan las opiniones de los participantes respecto a los caracteres anteriormente citados. En el parlicular, se muestran los matices de las respuestas de los participantes a aquellos ítems igualmente valorados y cuyas explicaciones revelan motivos diferentes.

\section{Estudio de un caso: mapa conceptual del profesor $\mathbf{A}$}

Nos parcce interesante mostrar, a modo de ejemplo y con cierto detalle, la aplicación de estos instrumentos al análisis de uno de los casos.
Presentamos el mapa conceptual integral del profesor «A» el cual nos servirá como punto de partida para el análisis que presentamos a continuación.

La tabla siguiente ha sido usada como resumen de los términos que aparecían en el mapa original del profesor «A» y cuyo sentido no era otro que explicitar los términos clave que aparecían en él. Los niveles a los que nos referimos en la tabla son un recurso que nos permite escribir los términos en el orden de lectura, de arriba hacia abajo, tal como fueron escritos inicialmente.

\begin{tabular}{|l|c|c|c|}
\hline & & E.D. & \\
\hline Álgebra & Análisis & Matem. & Aplicada \\
\hline Matemática & T. Cualitat. & T. Cuantit. & Modelizac. \\
\hline Conocim. & Empírico & Ciencias & Experimen. \\
\hline Filosofía & & Naturaleza & \\
\hline
\end{tabular}

\section{Microanálisis}

Algunos de los aspectos que resaltamos son:

- No se observa un orden jerárquico preestablecido, sino más bien una serie de ideas que conectan con la de ecuación diferencial. Relaciona las ecuaciones diferenciales con otras áreas de las matemáticas: análisis, álgebra, matemática aplicada y modelización.

Hice líneas, pero me volví loco... ¡Clarot, en un momento baja, en otro parece subir... porque tampoco hay una jerarquía clara...

(Entrevista profesor A)

- Establece una conexión entre el «modo de pensar matemático» y el «modo de pensar empírico»:

[...] la comprensión de las matemáticas está muy relacionada con la matemática aplicada. $Y$, por tanto, con el contacto con la situación en concreto. No tanto como algo que uno se abstrae, o sea, eso que tlaman matemática pura [...] Pienso que el empirismo es una de las formas del pensamiento matemático. Digamos así, sobre todo cuando viene desde esta cadena descendente: matemática, empirismo y filosofía.

(Entrevista profesor A)

- El término modelización le lleva a analizar la importancia del papel del especialista que interpreta el modelo y la dificultad de la búsqueda de expresiones matemáticas concretas.

El modelo suele ser muy complejo, y eso no hay quicn lo aborde. Se deja a los especialistas para que lo interpreten y hagan sugerencias del modelo, que ya tiene una expresión matemática concreta. Puede ser que este especialista no sea un 


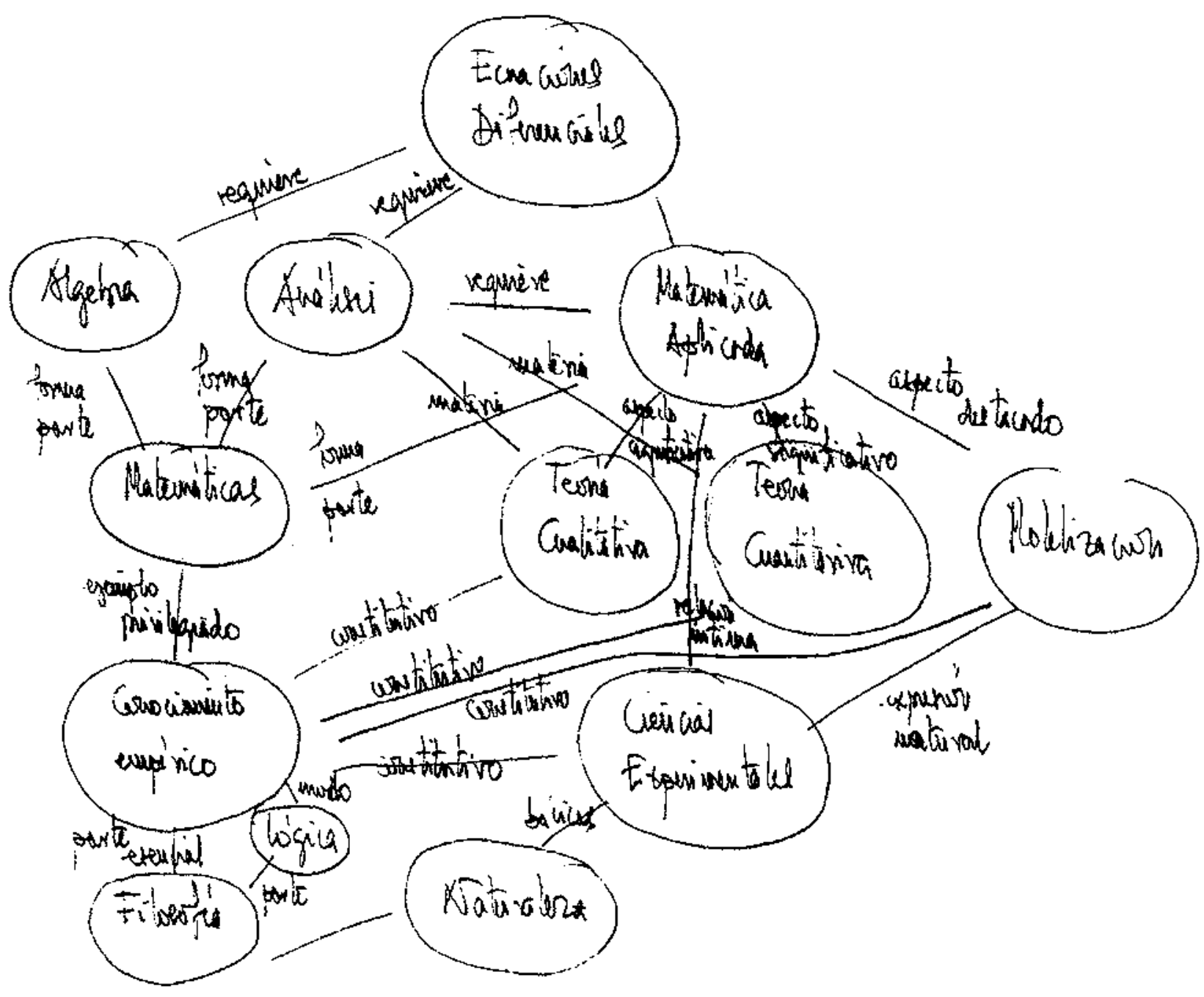

«fino especialista» en la parte de las matemáticas en las que en concreto ha caído ese modelo. En cualquier caso, muchas veces lo que se va haciendo es destacar de todas las intervenciones que hay en ese modelo las que son, por lo menos en unas aproximaciones iniciales, las más fundamentales. Entonces, un especiatista en modelización lo que tiene que intentar saber es, de las cosas que influye, digamos así, pues.. . ¿qué es lo que en un primer abordaje, en una primera forma de atacarlo, sería más interesante, y luego... pasar a él.

(Entrevista profesor A)

- La interpretación del modelo, según se haga desde las matemáticas o desde otros campos del conocimiento, hace variar ei discurso del profesor, produciendo situaciones conflictivas e intromisiones, no siempre adecua- das ni deseables, en un campo de conocimiento que no es el de uno mismo.

Aquí la motivación se queda en el modelo, aunque ilustramos con algunos modelos la situación matemática que estamos abordando, immm...! se quedan ahí... porque no podemos desarrollar el modelo estrictamente matemático. Aquí dar el modelo tampoco tiene mucho sentido porque... ¿cómo sé yo, primero, qué química se ve; 0 , cómo está de relacionado el modelo concreto con el que yo estoy buscando la ecuación, con Ios conocimientos que élos están dando? Además, puede ocurrir que, por no meterme en berenjenales, de los que a 30 mejor desconozco, resulte que estoy dando un modelo simplificado que ellos en ese momento a to mejor ya lo saben; y te quedas en una situación un poco perpleja, ¿no?

(Entrevista profesor A) 
- La valoración đe estas dificultades, añadida a la situación, al ambiente, a los objetivos del curso y a las difícultades de los estudiantes para asimilar la información que se les proporciona en un momento determinado, condiciona la actuación del profesor, lo cual hace desechar de sus programas los aspectos de modelización y centrarse más en un tratamiento procedimental de las ecuaciones diferenciales.

[...] aunque tiramos un poquito del modelo, nosotros no podemos tirar mucho; más que nada por el objetivo en el que se enmarca una asignatura como ésta. A este nivel, las matemáticas se toman de forma subsidiaria; Ios estudiantes hacen acopio de herramientas técnicas y se fuerza el aprendizaje de estas técnicas.

(Entrevista profesor A)

- Considera muy importante la relación de matemáticas y ciencias experimentales, como reflejan sus palabras:

Una ciencia progresa en la medida que se matematiza. Aunque no está puesta de forma directa esta relación, es evidente que cstá ahí. Ell sentido con el que digo eso es porque encauzan algo abstracto o etéreo de una forma más concreta.

(Entrevista profesor A)

\section{Macroanálisis}

En el mapa conceptual se observan dos ramas muy claramente diferenciadas:

a) una que unifica los conceptos más particulares en torno a las matemáticas;

b) otra que se diversifica a partir de la matemática aplicada.
Una primera mirada al mapa conceptual refleja:

Un primer zoom del mapa conceptual nos permitirá aunar «conceptos» y llegar a un «esqueleto» del mapa conceptual con un eje vertebrador central. Por un lado, la conexión cruzada entre matemática aplicada y matemática y, por otro, la de álgebra y análisis con matemáticas, permiten considerar un único nivel jerárquico formado por álgebra, análisis y matemática aplicada que confluyen en el término matemática.

[...] las ecuaciones han tenido tna cierta relación con otras partes, más o menos, de las matenáticas, en particular con el álgebra y el análisis. Éstos, at fin y al cabo, provienen de las matemáticas, pero tampoco las matemáticas tienen que ser algo alejado de la matemática aplicada. Lo que octurre es que separé lo que sería una relación rigurosa o matemática, de una relación en cuanto a su aplicabilidad, en cuanto a los modelos, o uno de los motores que ha tenido.

(Entrevista profesor A)

A partir de ahí, se continúa la cadena descendente que incluye, entre otras, Ias ciencias experimentales:

[...] la matemática «forma parte» (quizá aqui no lo puse, pero también podrías haber puesto en esta cadena, o quizás haberlo puesto yo)... como una parte del conocimiento empírico y, a su vez, el conocimiento empírico, digamos así... como una... de las actividades esenciales de la filosofía.

(Entrevista profesor $\mathrm{A}$ )

En virtud de la relación que se establece entre el conocimiento empírico y las ciencias experimentales, unificamos ambos términos en uno sólo que reza estudio de la realidad física. Más aún, el término filosofía lo englobamos en uno que diga naturaleza y el mundo; de esta forma, el mapa conceptual tendría ahora este aspecto:

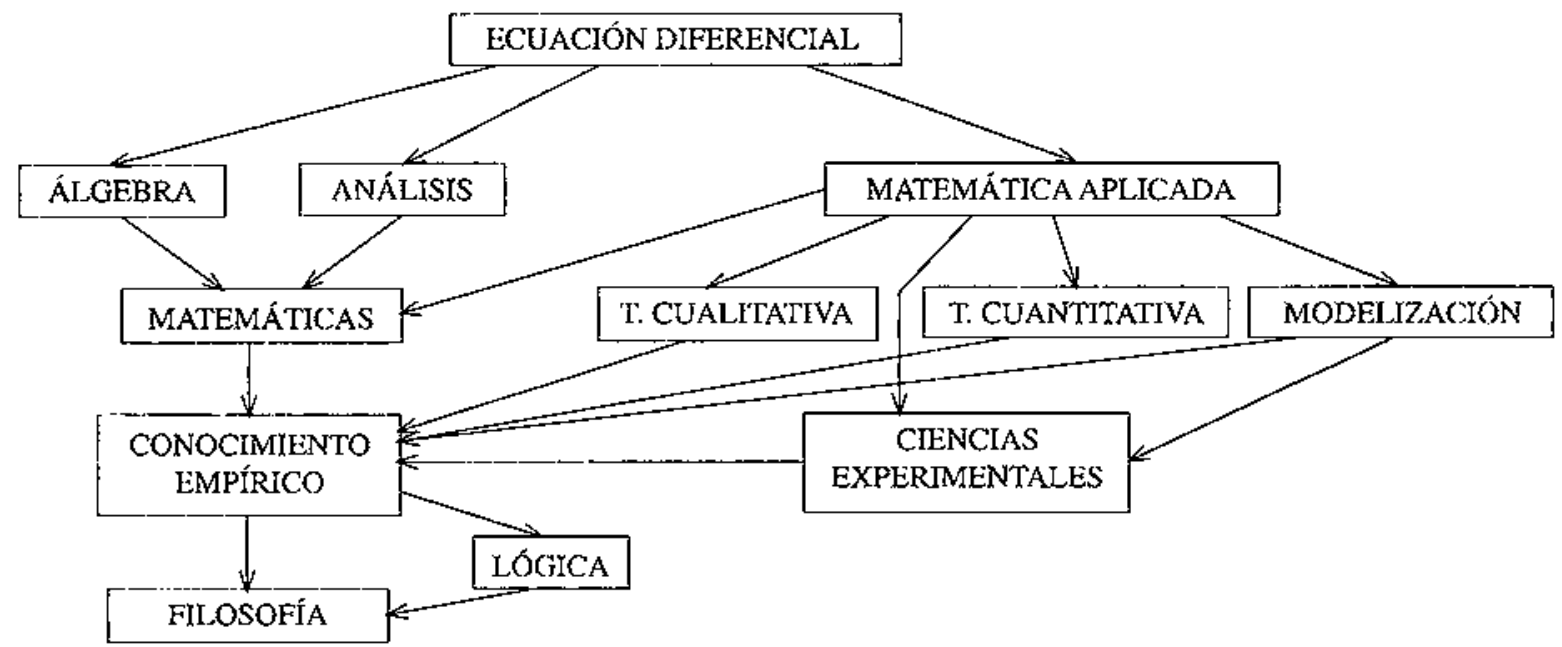


Mapa 3

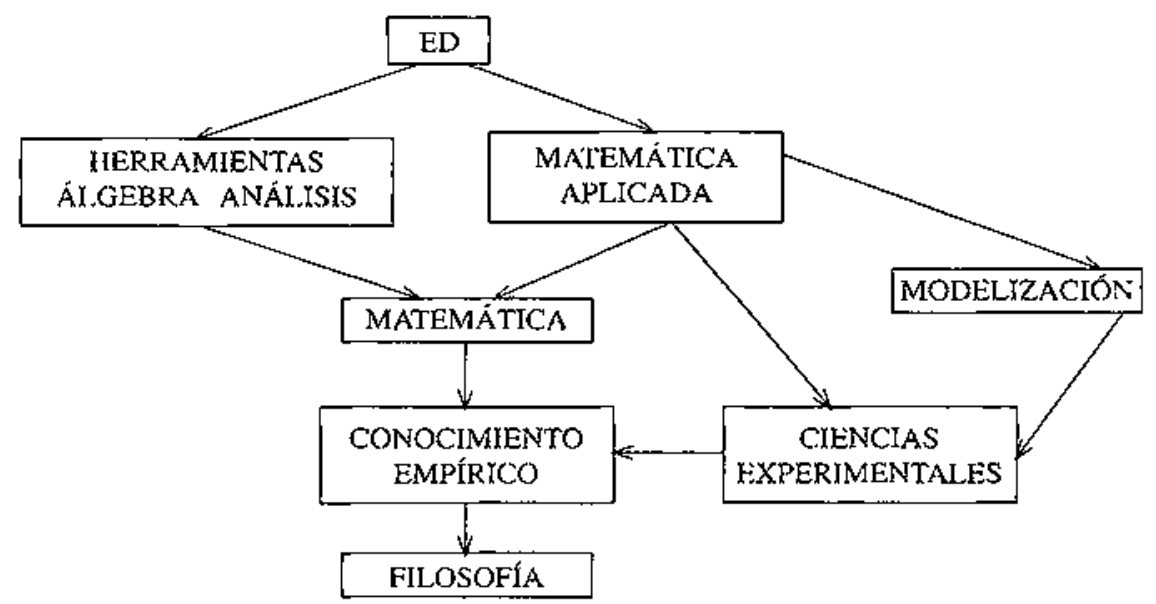

Mapa 4

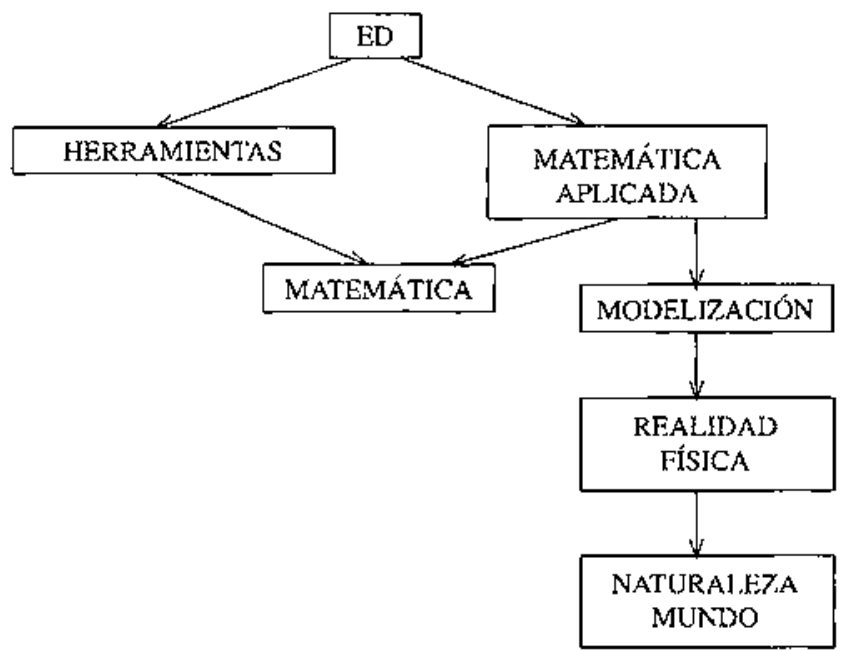

[...] es dentro del conocimiento un poco de filosofía, «los amigos del saber», de donde viene una gencalogía de estas cosas. O sea, que dentro de un motor que sería la curjosidad por el saber, pues está una de las cosas, el encauzar los razonamientos; [...] ho que ocurre es que dentro del saber hay muchas formas de encauzaslo y una de clias es lo que normalmente es esa argumentación más o menos rigurosa de los encadenamientos del pensamiento, pues puede ser el modo este, que va diciendo, de la lógica.

Fl empirismo es una forma de pensar matcmáticamente, de encauzar los conocimientos que provienen de situaciones que uno va viendo en la naturaleza. Normalmente todo surge porque hay un problema o una preocupación, un algo que te está moviendo a... que te despiertá la curiosidad.

(Entrevista profesor A)
En esta tercera aproximación, los conceptos fundamentales son: ecuación diferencial, matemática pura, matemática aplicada, realidad del mundo físico, estudio del mundo y de la naturaleza.

Aplicando una vez más un zoom, observamos que las tres ideas fundamentales que subyacen a todo son: matemática, realidad física y estudio del mundo. Tras este análisis podríamos pensar que su concepción sobre las ecuaciones diferenciales parece estar bastante próxima a la de Newton y Leibniz, y ciertos matemáticos del siglo XVII, tos cuales trataban de conjugar dos aspectos muy importantes: las matemáticas y la realidad del mundo físico. De esta forma el mapa conceptual quedaría como sigue: 
Mapa 5

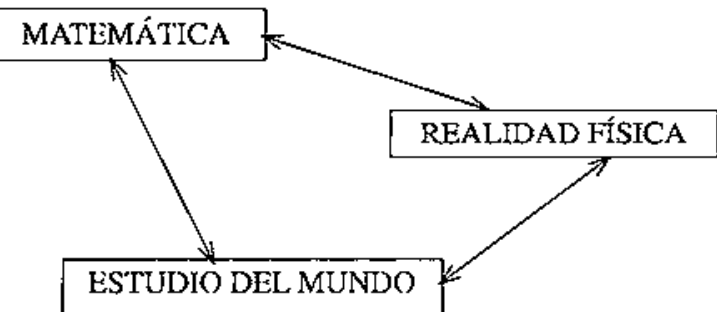

utilidad y sus aplicaciones; aquí la motivación se queda en el modelo, y no podemos tirar mucho de él.

(Entrevista profesor A)

- La práctica docente de este profesor acaba reducténdose a un acopio de algoritmos y técnicas de resolución de determinados tipos de ecuaciones diferenciales.

[...] a ti te dicen que tienes que dar un sumatio de técnicas y de herramientas sin motivarlas y sin encontrat la gracia que tiene todo eso.

(Entrevista profesor A)
Podríamos pensar, e incluso hipotetizar, que todas las ideas contenidas en el mapa conceptual originario quedarían reflejadas en este último.

\section{Conclusiones respecto a las concepciones del profesor $A$}

Daremos a continuación una relación de los rasgos que caracterizan las concepciones del profesor $\mathrm{A}$, a las que hemos Ilegado tras el análisis del mapa conceptual, la entrevista y materiales como exámenes y hojas de problemas:

- La globalidad y generalidad del mapa conceptual del profesor $\mathrm{A}$ es congruente con su interés personal y su curiosidad por las ciencias y las matemáticas, si bien este hecho no encuentra aplicación directa en su propuesta de trabajo a los estudiantes'

- La globalidad y generalidad observados en este profesor es un arma de doble filo que se vuelve contra él: por un lado, Ie permite ser flexible y tener una visión de conjunto de las matemáticas dentro de las ciencias experimentales y, por otro, le lleva a potenciar el enciclopedismo y mostrar las matemáticas como «objeto de interés, por y en sí mismo» independiente de otras áreas del saber.

[...] muchas veces nuestra falta de inteligencia o nuestra falta de comprensión de las cosas, porque no sabes entresacar de entre todas ellas las más relevanies. Muchas veces somos tercermundistas y queremos dar aquí la enciclopedia británica, ¡eso no deja de demostrar tu ignorancia ? [...] quizás el problema es nuestro y hay que enseñar mostrándoles los productos tal cual son y mostrándoles las ventajas que tiene para ellos.

(Entrevista profesor A)

- El tratamiento de la modelización resulta ser un punto de fricción entre lo que «piensa» y «lo que hace». No coincide la importancia concedida a este aspecto de las ecuaciones diferenciales en el mapa conceptual con el tratamiento posterior de cllo en el aula.

Los estudiantes están de aprendices y en este «taller» se les dice cómo se maneja el serrucho. Luego, más adelante ya les dirán por qué se maneja de esa forma y no de otra, cuál es la

\section{RESULTADOS DEL ANÁLISIS DE LOS DATOS}

\section{Los mapas conceptuales}

El primer análisis de los datos recogjdos de los cuatro profesores de la muestra desvela la existencia de tres concepciones diferentes.

Como hemos visto en el apartado anterior, en el mapa conceptual del profesor $\mathrm{A}$, las ecuaciones diferenciales aparecen como un bloque diferente del de álgebra y análisis; y aunque establece rclaciones y conexiones entre estos bloques de contenido, luego no las reflejaba en el aula. Proporciona un enfoque muy conceptual de las ecuaciones diferenciales y muestra lo que en sus palabras expresa como ...cierta tendencia al enciclopedismo...; es decir, una especie de inercia a dotar al estudiante de formalismos matemáticos y determinadas herramientas de trabajo sin importar demasiado cuál será su utilidad. Es como si, de alguna manera, se pensara que cuanto más contenidos se expliquen, más conocimientos tendrá el estudiante. Aunque es consciente de la necesidad de tener en cuenta las expectativas y necesidades de los estudiantes de ciencias experimentales y muestra flexibilidad para hacerlo, en la realidad cotidiana del aula estos intereses quedan de lado.

Los mapas conceptuales de los profesores $\mathrm{B}$ y C son muy similares, su estructura refleja un enfoque altamente procedimental de la materia y, en realidad, sus esquemas conceptuales responden más a cómo resolver tipos de ecuaciones diferenciales que a una idea general del concepto ecuación diferencial. Estos profesores muestran un firme convencimiento de la eficacia de la planificación de los temas:

[...] EDO de primer orden: son de este tipo... se resuelven de esta forma... pero se resuelven!, no se "prueba que se resuelven». A continuación tiene una lista de problemas muy extensa, no sé cúntos, pero, desde litego, 500 seguro.

Dedican muy poco tiempo a trabajar en problemas que modelizan situaciones reales:

Nosotros lo que hacemos es dar los métodos de forma abstracta, luego dedicas una o dos semanas, ¡nunca más de dos!, a explicar problemas como el del tanque que se llena de agua, 
calor en una habitación, etc. ¡Peroeso expresamente y dedicado a eso!

Justifican esta planificación y diseño de la asignatura como la consecuencia del bajo nivel de conocimiento de los estudiantes y el poco interés por la materia.

La práctica docente de los profesores A, B y C no difiere más en los elementos propios que, en to personal, aporta cada uno de ellos a su aula. En todos ellos se deja sentir claramente la influencia de un discurso elaborado desde las matemáticas y para matemáticos, con total ausencia de elementos que aludan a los estudiantes de ciencias experimentales.

El mapa conceptual del profesor D varía considerablemente del de los otros tres participantes en el estudio. Este mapa conceptual refleja todo un planteamiento de la asignatura al servicio de la formación de los estudiantes. Se equilibran los aspectos conceptuales y procedimentales. Los aspectos matemáticos trabajados se consideran los necesarios en cada momento y se justifican, siempre, desde situaciones contextualizadas de interés para este colectivo.

[...] puesto que de lo que se trata de abordar elementalmente es el estudio de las EDO y en diferencias como modelos para describir procesos propios de la biología de poblaciones, se hace referencia a ello desde el principio, evitando el tradicional sistema de cargar en formalismos el texto y acudir posteriormente a ejempios. Por lo tanto, lo importante aquí está en que el individuo tenga la idea clara y sepa usar las matemáticas como instrumento. [...] Para tratar de conseguirlo, se pretende un acercamiento al método de las motivaciones instrumentales, flexibilizando la expresión de referentes a situaciones concretas e inteligibles para poder conseguir un grado razonable de autocontenido.

(Entrevista profesor D)

Se intenta tener en cuenta el desarrollo cognitivo del sujeto, más que el desarrollo lógico de la propia materia. Se elabora un discurso para químicos o biólogos teniendo en cuenta sus necesidades futuras y sus expectativas.

Desde el punto de vista metodológico, creo que es un disparate empezar con el desarrollo matemático de las EDO para luego acabar poniendo ejemplos; siempre tratamos de presentar una situación en biología y traducirla al lenguaje matemático.

En cualquier ciencia experimental hay que enfocar las matemáticas de manera que se tengan muy claras las ideas y se sepan utilizar tales ideas, pero sin caer en las ortodoxias típicas de las facultades de matemáticas en las que se construye un discurso deductivo valorable en sí mismo y no tiene una intencionalidad exterior a la matemática. Por tanto, lo importante aquú eșé en que el individuo tenga la idea clara y sepa usar de las matemáticas como instrumento.

(Entrevista profesor D)

\section{Los cuestionarios}

Algunos de los resultados relativos a los aspectos más conceptuales, procedimentales y metodológicos provienen, en su mayor parte, del análisis de los cuestionarios.
Mostramos algunos de ellos de acuerdo con los caracte res ya eninciados.

\section{a) Respecto a los métodos de resolución}

Todos los participantes en el estudio declararon que el método de resolución de las ecuaciones diferenciales, conceptualmente bueno, era el algebraico o analítico. Sin embargo, los motivos que esgrimieron fueron variados:

- El profesor A no desdeña ningún método de resolución y recalca el interés de los métodos gráficos en el tratamiento de los problemas no lineales. No piensa que ninguno de los métodos entrañe una dificultad especial y afirma, por el contrario, que el profesor debería presentar a los estudiantes más situaciones variadas donde emplear diversos métodos de resolución. Estas opiniones se mueven en el terreno teórico, pues en la práctica casi ni hace referencia a los métodos de resolución gráficos.

En general cl método gráfico es una tontería, pero trae mucha información. Sobre todo tienen interés para los problemas no lineales. Dependiendo del problema, trataría de usar uno tr otro método. Veo todos los métodos sencilios.

(Entrevista profesor A)

- Los profesores B y C consideran que tanto los métodos analíticos como los numéricos son igualmente sencilios, aunque estos últimos casi ni se explican. No comentan nada de los métodos gráficos y además puntualizan que éstos no son lo «suficientemente matemáticos».

Sốlo vemos los métodos de resolución algebratcos, los numéricos no se ven en esta asignatura y los gráficos ni siquiera decimos que existen.

(Profesores B y C)

- La línea de trabajo elegida por el profesor D es la algebraica, aunque considera que los métodos numérico y gráfico deben ser un complemento del algebraico en aquellas situaciones donde el uso de los primeros dificulte la solución de un problema $o$, simplemente, cuando no sea posible resolverlo. En su caso particular, los estudiantes tienen la posibilidad de trabajar con los tres enfoques y en entornos de trabajo muy diferentes que incluyen el uso del ordenador.

Conceptualmente creo que cl método bueno es el algebraico. El métodio numérico, si no tienes bien trillado el planteamiento algebraico, creo que sería complicado. Desde el punto de vista metodológico y conceptual, el método numérico debe recurrirse como tal recurso, no como un planteamiento previo. El método gráfico se usa poco, parece que es un complemento para ver las cosas, pero no algo en sí mismo bueno.

(Entrevista profesor D)

\section{b) Respecto al objetivo y tipo de actividad propuesta}

Destacaremos los resultados más significativos:

- El profesor A es cauto, pues, aunque piensa que es más interesante plantear problemas con más «literatura», en 
vez de ejercicios o problemas de aplicación, hay que tener cuidado con las dificultades que pueden provocar una mala expresión de las condiciones del problema. Esto añade una dificultad a las propias de la tarea en sí misma. Sin embargo, el tipo de actividad propuesta suele ser de aplicación de métodos de resolución analíticos, en los que las dificultades surgen por el tipo de ecuación diferencial planteada. En raras ocasiones propone tareas puramente teóricas.

[...] cada vez más, en los exámenes intentamos que aparezca "más literatura"; pero hay que tener cuidado porque eso muchas veces noes tan sencilto como parece, ya que los datos, los tienes que dar que sean sugerentes, pero que, por otra parte, sean unívocos de su tratamiento, ia veces se dan con una ambigivedad...!

(Entrevista profesor A)

- El profesor B piensa que la actividad matemática, sea cual sea, tiene valor «en sí misma», independientemente del contex to. Lo que importa es que el estudiante demuestre que ha aprendido unos conocimientos matemáticos y maneje con destreza los métodos de resolución sistemáticos. Por este motivo, el tipo de actividad propuesta en clase, exámenes y hojas de problemas son ejercicios de aplicación inmediata de cualquiera de los métodos de resolución, previamente explicados en la clase.

[...] considero que los automatismos y la rutina es importantísima. Barece ser que la rutina es un término peyorativo pero, para mí, en esta asignatura es fundamental, porque precisamente es to que estamos inculcando; equivocados o no, pero es así.

(Entrevista profesor B)

- La opinión del profesor C es muy próxima a la del profesor $\mathrm{B}$, aunque añade que cada profesor debería hacer un esfuerzo por buscar y proponer a los estudiantes tareas que provocaran el interés y motivación de éstos. En este sentido, este profesor busca variaciones a los ejercicios rutinarios mediante cambios numéricos, incorporación de parámetros a las ecuaciones, etc.

[...] e] caso es que nosotros to hacemos siempre así, presentando sistemas y que lo restueivan sin ponerto dentro de una situación; pero me parecería más interesante que el sistema respondiera a algo real.

(Entrevista profesor C)

- El profesor D se inclina por actividades contextualizadas y que respondan a situaciones reales o de laboratorio, frente a las cuales puedan estar un día los futuros químicos y biólogos. En particular son importantes aquéllas que impliquen modelos exponenciales o asimilados a ellos. En contadas ocasiones propone alguna actividad exclusivamente sistemática y rutinaria; sin embargo, la dinámica general es la de proponer situaciones problemáticas contextualizadas y que permita a los estudiantes aplicar los conocimientos explicados por el profesor.

Se establece previamente un modelo, por ejemplo, la tasa de crecimiento instantánea; ésta viene asociada a un modelo, el tipo de ecuación puede responder a una integración rápida... pero en ningún momento les pedimos que sepan diferenciar típos de EDO y sepan resolverlas de acuerdo con métodios tipiftcados; aún más, nunca sc lo contamos. Está siempre unido a aquello que son los intereses y motivaciones de los alumnos.

[...] Me parece una barbaridad proponer ejercicios rutinarios, y me parece que nodeben plantearse así.El objetivo probablemente es que la gente aprenda eso, pero hay que expresarlo en su lenguaje. Si pretendes que la gente aprenda a modelizar, tienes que cnseñarle a aprender a traducir, al lenguaje matemático, una realidad.

(Entrevista profesor D)

Finalmente, los profesores A, B y C valoran positivamente el uso de ejercicios rutinarios y de aplicación directa de métodos, ya que es una forma de aprender a manejar la herramienta que se les presenta. Además, desde el punto de vista de la evaluación, les resulta más sencitlo de evaluar. El profesor D no es partidario del uso abusivo de estos ejercicios, aunque piensa que siempre es importante ser consciente de la parte de sistematicidad que conllevan algunos procedimientos matemáticos. Por tal motivo, en sus hojas de problemas aparecen algún que otro ejercicio rutinario y sistemático con el único objetivo de que los estudiantes conozcan la existencia de métodos sistemáticos y adquieran cierto manejo de éstos.

\section{c) Respecto al conocimiento de la historia}

En este aspecto se distinguen claramente dos tendencias:

- Los profesores A y D conceden mucha importancia a los conocimientos, por parte de los profesores, de la historia de las matemáticas y evolución de los conceptos. El regreso a las fuentes es un referente que permite buscar alternativas conceptuales a hechos que, explicados desde el lenguaje aigebraico y la teoría de conjuntos que envuelven toda la matemática, se hacen difíciles y farragosos de entender. De hecho, ambos profesores tienen en cuenta la historia en la elaboración y preparación de los temas del programa.

- Los profesores B y C se quedan en el conocimiento anecdótico de determinados episodios de la historia y no conceden mayor importancia a ésta, más que como posible elemento motivador para los estudiantes. En ningún caso, estos profesores hicieron referencia al uso de la historia como fuente de información en la preparación de los temas.

\section{d) Respecto al uso de nuevas tecnologias}

Otro aspecto destacado, en este análisis inicial de los resultados, es que, salvo el profesor $\mathrm{D}$, ninguno de los restantes participantes en el estudio incorpora el ordenador u otras tecnologías como instrumento de enseñanzaaprendizaje. Mientras que el profesor A piensa que seráa interesante y necesaria la incorporación de los ordenadores al ámbito universitario, reconoce las limitaciones del profesorado y apunta al tiempo y trabajo requerido para organizar, điseñar y preparar prácticas de interés que justifiquen el uso del ordenador. Los profesores $B$ y C sólo ven el ordenador como un elemento motivador que no cambiaría en gran medida el actual planteamiento de la asignatura. 
Tratamos de ver que hay que recurrir a otras cosas cuando éstas no funcionan bien. Se usa fundamentaimente el «derive»; a veces, las ED se resuelven en prácticas y la mismá vuelve a resolverse en el ordenador para que puedan ver la diferencia de complejidad y la rapidez de acceso al usar un paquete informático.

(Entrevista profesor D)

Sería darle un poco la vuelta a lo que estamos dando, porque, los contenidos, creo que serían los mismos, pero... sería la presentación lo que habría que cambiar y la motivación.

(Entrevista profesor C)

\section{CONCLUSIONES}

\section{Conclusiones metodológicas}

El carácter exploratorio de nuestro estudio nos ha lleva. do a diseñar y aplicar la metodología que consideramos ad hoc. Nuestras conclusiones en este sentido son las siguientes:

- La riqueza de los mapas conceptuales junto con las explicaciones grabadas de cada profesor han proporcionado gran variedad de detalles sobre cada uno de ellos, lo que nos ha permitido tener una descripción bastante detallada de cada participante acerca de sus concepciones de la enseñanza de las ecuaciones diferenciales; además, nos reafirmamos en la importancia del análisis de mapas conceptuales siempre contrastado con la interpretación hecha por el autor.

- El análisis del mapa conceptual en dos niveles ha sido muy valioso y eficaz. Por un lado, el microanálisis ha permitido adentrarnos, con cierto lujo de detalles, en algunas de las concepciones de los participantes sobre la enseñanza de las ecuaciones diferenciales y acceder a detalles muy concretos que, de otro modo, habrían pasa do inadvertidos. A su vez, el macroanálisis ha proporcionado ideas muy generales sobre aspectos primordiales de la enseñanza y aprendizaje de las matemáticas, a los que cada profesor concede especial importancia.

- Por su parte, el cuestionario se reveló como un buen instrumento de validación de los mapas conceptuales.

- A pesar de la dificultad del análisis y codificación de los datos, creemos que la metodología diseñada para la investigacion ha resultado un buen instrumento de recogida y análisis de datos en una investigación de las características de la que presentamos aquí.

\section{Conclusiones generales del estudio}

Las conclusiones son fruto de la reflexión apoyada en el marco teórico elegido como modelo de explicación de los hechos observados, detectados y descubiertos. Hemos estructurado las conclusiones de acuerdo con los objetivos planteados inicialmente: a) Indagar acerca de las concepciones de los profesores de matematicas sobre la enseñanza de las ecuaciones diferenciales en carreras científico-experimentales

Se trataba de analizar, con la mayor exactitud posible, las actitudes, creencias y sistemas de valores de los profesores frente al contenido matemático, la profesión docente y los estudiantes. El análisis de los datos nos sugirió tipificar los rasgos metodológicos, didácticos y cognitivos de los profesores participantes, en estructuras que nos permitieran caracterizar algunos estilos de enseñanza vigentes en la universidad.

Las respuestas de los profesores han determinado tres estilos diferentes, que van desde aquél que mantiene un tratamiento más estructural de las ecuaciones diferenciales y de las matemáticas hasta aquél que, en el otro extremo, propone un planteamiento metodológico muy próximo a los intereses de las ciencias experimentales y considera las ecuaciones diferenciales como el instrumento para resolver problemas químicos o biológicos. En el centro de esta escala, pero extendiendo su dominio de existencia en un entorno bastante amplio a su alrededor, estaría aquel estilo en el cual el profesor entra en conflicto entre «lo que hace» y lo que «se podría hacer», es decir, está en transición. Estos estilos, los denominamos: tradicional, transitorio y avanzado.

Brevemente, daremos algunas de las características de los estilos docentes definidos:

\section{Estilo tradicional}

- Potencia el aspecto procedimental sobre el conceptual. - Toma la matemática como "objeto en sí misma».

- Los problemas de modelización son usados como ejemplos.

- Se tiende al acopio de técnicas y métodos de resolución analíticos.

- Se elabora un discurso matemático para matemáticos. - Hay ausencia del ordenador en el aula como instrumen. to de aprendizaje.

- La enseñanza no es globalizada.

\section{Estilo transitorio}

Lo que diferencia a uno del otro es que el profesor enmarcado dentro del estilo transitorio, por sus conocimientos científicos, ideas acerca de la materia, desarroIlo profesional e interés y curiosidad personal, es susceptible de pertenecer al estilo avanzado; sin embargo, entra en contradicción consigo mismo en cuanto a la práctica docente, la cual se aproxima al estito tradicional.

\section{Estilo avanzado}

- Se integra la matemática con las situaciones reates y la biología.

- Encuentra un equilibrio entre las dos dimensiones de la matemática: como objeto y como instrumento. 
- Los problemas de modelización se integran de forma natural en el curriculo.

- Se motivan la enseñanza de las ecuaciones diferenciales a partir de modelos biológicos.

- Se clabora un discurso matemático para biólogos.

- Se incorpora el ordenador como instrumento de enseñanza-aprendizaje.

- La enseñanza de las ecuaciones diferenciales es globalizada.

\section{b) Detectar y descubrir dificultades relativas al "qué»y "cómo» enseñar las ecuaciones diferenciales en dichas titulaciones}

Los estilos de enseñanza que hemos asociado a cada profesor del estudio parecen incumplir ciertos principios básicos, cognitivos y didácticos, lo que desde nuestro punto de vista podría propiciar obstáculos que dificultaran el desarrollo adecuado del proceso de enseñanza-aprendizaje. No obstante, queremos hacer hincapić en el hecho de que muchas de las conclusiones a las que llegamos en este estudio han sido tomadas como hipótesis y punto de partida para la investigación que actualmente está en marcha.

Atendiendo a los aspectos cognitivos podemos observar que los estilos tradicional y transitorio centran su enseñanza más en el aspecto del proceso, haciendo acopio de técnicas de resolución de ecuaciones diferenciales; de esta forma, conjeturamos que los estudiantes se convertirían en hábiles resolutores de ecuaciones diferenciales y desarrollarian de una forma incompleta el procepto ecuación diferencial.

La flexibilidad de pensamiento sería pequeña, lo que produciria esquemas conceptuales muy pobres. Debido a esta incompletitud y escase $z$ del sistema de representación proceptual, los estudiantes serían incapaces de generar una variada gama de representaciones mentales asociadas al concepto.

Nuestra hipótesis es que estos hechos parecen reducir el número de conexiones entre esquemas, con lo cual la tensión cognitiva sería alta y la recuperación de la información se haría bastante difícil. La respuesta de muchos estudiantes a esta incapacidad para manipular un concepto, o enriquecer el esquema conceptual existente con nueva información, se traduciría en rutinizar las secuencias de actividad, lo que explicaría, en parte, ej alto fracaso de las matemáticas en las facultades de ciencias experimentales.

Por otro lado, el estilo avanzado parte del planteamiento de las ecuaciones diferenciales como procepto, en cuanto que son «unos objetos matemáticos» y «unos instrumentos fundamentales» para conducir formalmente los modelos determinísticos continuos. Desde un principio (en palabras del profesor D), los estudiantes manejan las ecuaciones diferenciales asociadas a modelos, en referencia a funciones, en relación con la tasa de variación, como derivada-antiderivada, etc., con lo cual pensamos que se enriquecerían sus sistemas de representación y se produciría algo así como una red de esquemas concep- tuales, cada vez más complejos, asociados a la noción de ecuación diferencial.

En este caso, nuestra hipótesis es que las conexiones entre esquemas conceptuales existentes serían múltiples, lo que maximizaría la recuperación cognitiva y flexibilizaría la visión proceptual de las matemáticas. Esta flexibilidad del pensamiento en este estilo avanzado vendría potenciada por la manipulación simultánea que el estudiante hace de las representaciones gráficas, numéricas y simbólicas.

Desde un punto de vista didáctico, podemos decir que el estilo avanzado es un ejemplo de modelo de enseñanza basado en la búsqueda de situaciones didácticas, a través de modelos biológicos, reales o diseñados en el laboratorio por los profesores, que enfrentarían a los estudiantes a situaciones «reales» que encontrarán fuera del contexto de la clase de matemáticas.

Los estilos tradicional y transitorio no son tan ricos en la motivación de este tipo de situaciones (aunque en el transitorio pueden producirse alguna vez). Por el contrario, las dificultades y obstáculos, tanto para la enseñanza como el aprendizaje, son considerables, lo que lleva a los profesores a establecer «implícitamente» un contrato didáctico "adecuado" para las dos partes implicadas. De esta forma, se reducen contenidos, se proponen ejercicios muy procedimentales y rutinarios, los exámenes se asimilan en un $80 \%$ o $90 \%$ a los ejercicios vistos en clase; se hace un asignatura basada en reglas. La consecuencia de esto suele contar con el beneplácito de ambas partes:

- los profesores no tienen sensación de fracaso;

- Los estudiantes tienen sensación de aprender algo nuevo y la posibilidad de destacar, cuando no de aprobar con facilidad.

Es propio de los estilos tradicional y transitorio fenómenos como el del deslizamiento metadidáctico, que consiste en transformar lo que en un primer momento fue medio de enseñanza en objeto de estudio e incluso, en ocasiones, en objeto de enseñanza: la forma substituye el fondo (Brousseau, 1990). De este modo, sucede que el profesor tiene que justificar y legitimar, ante sí mismo, la institución y la sociedad, las modificaciones de cláusulas del contrato. Así, a menudo alude al poco tiempo del que se dispone, al bajo nivel de los estudiantes, a la extensión de los programas, etc.

Aceptando que todos los condicionantes a los que acabamos de aludir son ciertos, el profesor de estilo avanzado intentaría evitarlos trabajando desde una propuesta $\mathrm{cu}$ rricular "al servicio de los estudiantes" (es decir, de su formación) y con una línea metodológica que motivara a los estudianies y donde los objetivos estarían perfecta. mente claros. El éxito del proceso de enseñanza en el estilo avanzado residiría en:

- explicitar desde el principio, muy claramente, Ios objetivos que se pretenden lograr; 
- tener en cuenta a los receptores del discurso; - ser natural, traducir el lenguaje matemático a su realidad, siendo extremadamente riguroso y claro.

En el caso de los estilos tradicional y transitorio, la enseñanza consiste en una traducción directa del formalismo matemático habitual, acompañado en ciertas ocasiones de un intento de reduccionismo de ta complejidad cognitiva de aspectos matemáticos. Esto acompañado de la fragilidad y pobreza de estructuras cognitivas explica* ría la situación de "fracaso» a la que conducen estos modelos đe enseñanza muy próximos a los que tradicio- nalmente han ocupado la práctica totalidad de las instituciones de enseñanza.

\section{NOTA}

1 Esta afirmación proviente de la información directa del profesor, como consecuencia de contraponer sus ideas personales sobre la materia y el tipo de actividades propuestas a los estudiantes. En ningún momento se ha hecho observación de aula, por lo que algunas de nuestras conclusiones pueden ser tomadas como hipótesis de trabajo, sobre las que profundizar, para el trabajo de tesis que está en marcha.

\section{REFERENCIAS BIBLIOGRÁFXCAS}

ARTIGUE, M. (1989). Une recherche d'ingénierie didactique sur l'enseignement des equations différentielles en primer cycle universitaire. Cahiers du Séminaire de Didactique des Mathématiques et de l'Informatique de Grenoble. Grenoble: IMAG

AZCÁRATE, C. (1990), La velocidad introducción al concepto de derivada. Tesis de doctorado. Universitat Autònoma de Barcetona.

AZCÁRATE, C. (1992). Estudio de los esquemas conceptuales y de los perfiles de alumnos de $2^{\circ}$ de BUP, en relación con el concepto de pendiente de una recta. Epsilón, 24.

BROUSSEAU, G. (1988). Le contrat didactique: te milieu. Recherches en Didactique des Mathématiques, 9(3), pp. 309-336.

BROUSSEAU, G. (1990). ¿Qué pueden aportar a los enseñantes los diferentes enfoques de la didáctica de las matemáticas? (Primera parte). Enseñanza de las Ciencias, $8(3)$, pp. $259-267$.
CHEVALLARD, Y. (1991). La transposition didactique. Du savoir savant au savoir enseigné. Un exemple de la transposition didactique. Grenoble: La Pensée Sauvage.

DUBINSKY, E. y LEWIN, P. (1986). Reflective abstraction and mathematics education: The genetic decomposition of induction and compactness. Joumal of Mathematical Behavior, 5(1), pp. 55-92.

DREXFUS, T. (1990). Advanced Mathematical Thinking, en Nesher y Kilpatrick (eds.). Mathematics and Cognition, pp. 113-134. ICMI Study Series. Cambridge University Press.

DREYFUS, T. (1994). Imagery and Reasoning in Mathematics and Mathematics Education, en Selected Lectures from the VII International Congress on Mathematics Education. Québec, Canads: Les Presses de L'Université Laval.

MILES, M.B. y HUBERMAN, A.M. (1987). Data Analysis. A Source of New Methods. Beverly Hitls: Sage Publications. 
MORFNO, M.M. (1995). Enseñanza de las ecuaciones diferenciales a químicos y biólogos desde la perspectiva del profesor de matemáticas. Estudio de casos. Tesis de maestría. Programa de Doctorat del Departament de Didactica đe la Matemàtica y de les Cièncias Experimentals, Universitat Autònoma de Barcelona.

NOVAK, J.D y GOWIN, D.B. (1988). Aprendiendo a aprender. Madrid: Martínez Roca.

SFARD, A. (1987). Two Conceptions of Mathematical Notions: Operational and Structural, en Proceedings of the Eleventh International Conference of PME, Montreal, 3, pp. 162169.

SFARD, A. (1991). On the dual nature of mathematical conceptions: reflections on processes and objects as different sides of the same coin. Educational Studies in Mathematics, 22, pp. 1-36.

TALL, D.O. y VINNER, S. (1981). Concept image and concept definition in Mathematics with particular reference to limits and continuity. Educational Studies in Mathematics, 12, pp. 151-169.
TALL, D.O. (1994a). A Versatile Theory of Visualisation and Symbolisation in Mathematics. Plenary Presentation at the Commission Internationale pour l'Étude et l'Amélioration de l'Enseignement des Mathematiques, Toulouse, Francia, julio I994, pp. 1-12.

TALL, D.O. (1994b). Understanding the Processes of Advanced Mathematical Thinking. Han invited ICMI lecture at the International Congress of Mathematicians, pp. 1-9. Zurich.

TALL, D.O. (1995). Cognitive Growth in Elementary and Advanced Mathematical Thinking. Plenary Lecture at the Annual Conference of the International Group for the Psychology of Mathematics Education, pp. 1-9. Recife, Brasil, juhio de 1995.

VINNER, $S$. (1989). The avoidance of visual considerations in calculus students. Focus on Learning Problems in Mathcmatics, 11(2), pp. 149-155.

VINNER, S. y DREYFUS, T. (1989). Images and definitions for the concept of function. Joumalfor Research in Mathematics Education, 20, pp. 356-366

[Artículo tecibido en febrero de 1996 y aceptado en diciembre de 1996.] 